\title{
Effects of tryptophan depletion on anxiety, a systematic review
}

\author{
Simone M. E. Schopman (1) ${ }^{1,2}$, Renske C. Bosman (1) ${ }^{1,2}$, Anna D. T. Muntingh ${ }^{1,2}$, Anton J. L. M. van Balkom ${ }^{1,2}$ and \\ Neeltje M. Batelaan ${ }^{1,2}$
}

\begin{abstract}
Vulnerability markers for onset of anxiety disorders are scarce. In depression, patients at risk tend to respond with a negative mood to 'acute tryptophan depletion' (ATD), while healthy volunteers and current patients do not. The serotonergic system thus provides indications for vulnerability for depression. It is unknown whether ATD reveals vulnerability in anxiety too. This study systematically reviews the effects of ATD on anxiety and assesses whether challenging anxiety modifies the response. PubMed, Embase and Psychlnfo were systematically searched up to April 2019 for studies in which (1) healthy volunteers or patients with a (remitted) anxiety disorder underwent ATD and (2) levels of anxiety were reported. In total, 21 studies were included. Studies conducted in healthy volunteers $(n=13)$, and patients with a remitted $(n=6)$ or current (panic, social or generalised) anxiety disorder $(n=4)$. Studies were mostly of poor quality and heterogeneous regarding population, challenge test used and outcome measures. ATD did not consistently affect anxiety in any of the groups. Moreover, a challenge test after ATD ( $n=17$ studies) did not consistently provoke anxiety in healthy volunteers or remitted patients. A $35 \% \mathrm{CO}_{2}$ challenge did consistently increase anxiety in patients with a current panic disorder (PD). To conclude, this systematic review found no clear indications that ATD provokes anxiety in those at risk for anxiety disorders. Hence, unlike in depression, ATD does not indicate vulnerability to develop an anxiety disorder. Because included studies were heterogeneous and mostly of poor quality, there is an urgent need for high quality research in homogeneous samples.
\end{abstract}

\section{Introduction}

Anxiety disorders are severe and disabling disorders and many patients have a poor course trajectory ${ }^{1-4}$. Although sociodemographic and clinical factors have been associated with developing anxiety disorders (e.g. gender, disability $)^{5,6}$, their predictive validity is low. Studies of biological factors (e.g. hypothalamic-pituitary-adrenal axis functioning, inflammation markers) show inconclusive results ${ }^{7-10}$. Currently, vulnerability to develop an anxiety disorder cannot be revealed.

In depressive disorders, the serotonergic (or 5hydroxytryptamine [5-HT]) system provides an indication

\footnotetext{
Correspondence: Simone M. E. Schopman (s.schopman@ggzingeest.nl) 'Amsterdam UMC, Vrije Universiteit, Department of Psychiatry, Amsterdam Public Health institute, Amsterdam, The Netherlands

${ }^{2}$ GGZ inGeest Specialized Mental Health Care, Amsterdam, The Netherlands

These authors contributed equally: Simone M. E. Schopman, Renske C. Bosman
}

for vulnerability. This neurotransmitter system is involved in the regulation of emotions and seems dysregulated in depressive disorders ${ }^{11}$. The serotonin system in depression has been investigated repeatedly by lowering serotonin levels with $\mathrm{ATD}^{12}$. In ATD, the availability of the essential amino acid tryptophan, the dietary precursor of serotonin, is decreased by means of a specifically designed amino acid drink. ATD is assumed to result in a decrease of the availability of serotonin in the central nervous system ${ }^{13}$ (see Methods for more details).

Responding to ATD with a lower mood seems to reveal vulnerability for developing a depressive episode. A metaanalysis showed that in healthy volunteers without a family history of depression no changes in mood occurred $^{14}$. However, in persons at risk for depression, including healthy volunteers with a positive family history and remitted depressed patients, a decrease in mood did

\section{(c) The Author(s) 2021}

(c) (i) Open Access This article is licensed under a Creative Commons Attribution 4.0 International License, which permits use, sharing, adaptation, distribution and reproduction cc) in any medium or format, as long as you give appropriate credit to the original author(s) and the source, provide a link to the Creative Commons license, and indicate if changes were made. The images or other third party material in this article are included in the article's Creative Commons license, unless indicated otherwise in a credit line to the material. If material is not included in the article's Creative Commons license and your intended use is not permitted by statutory regulation or exceeds the permitted use, you will need to obtain permission directly from the copyright holder. To view a copy of this license, visit http://creativecommons.org/licenses/by/4.0/. 
occur, with the effects being more prominent for remitted patients. Moreover, a deterioration of mood after ATD in remitted depressed patients predicted a higher chance to develop a new depressive episode ${ }^{15}$. In current depressed patients no mood response was observed ${ }^{14}$, probably because the disorder is already present ${ }^{16}$, and thus there is no risk for developing a depression.

The serotonergic system also plays a role in the pathogenesis of anxiety disorders ${ }^{11,17}$. A positron emission tomography study showed that compared to controls, patients with social anxiety disorder (SAD) had an overactive presynaptic serotonin system. Increased serotonin synthesis and serotonin transporter availability was observed in several brain regions ${ }^{18}$. Serotonin is also the mode of action of antidepressants, which are effective in treating anxiety disorders ${ }^{19,20}$. Details of the underlying mechanisms of the role of the serotonin system in anxiety disorders are still unknown. Given that ATD seems to reveal vulnerability in depressive disorders and the involvement of the serotonergic system in both disorders, ATD could potentially reveal vulnerability for developing anxiety disorders.

The first aim of this study was to systematically review the effects of ATD in healthy volunteers and patients with a remitted or current anxiety disorder on a change in reported levels of anxiety. Provided that ATD reveals vulnerability in anxiety disorders, it is expected, in line with the findings for ATD in depression ${ }^{14}$, that for healthy volunteers ATD will have little effect on anxiety, that ATD will increase anxiety in remitted patients, and that no effect will be found of ATD on anxiety in current patients.

A difference between depressive disorders and anxiety disorders is that a negative mood in depressive disorders seems more constantly present, whereas anxiety occurs predominantly when a patient is exposed to anxietyprovoking situations. Therefore, anxiety might need to be challenged. The second aim of this study is, thus, to review whether provoking anxiety by means of a challenge test modifies the anxious response after ATD. The challenge test is expected to increase anxiety independent of ATD. It is expected that using a challenge test after ATD will result in a more prominent anxious response.

\section{Methods}

\section{Literature search}

PubMed, Embase and PsychInfo were searched by an experienced librarian and SMES from inception to April 2019. The search string consisted of (combinations of) free text and keywords indicative for anxiety disorders and tryptophan depletion (search strategy in Appendix 1). Language was unrestricted. The search was extended by scanning the references of the included articles.

Inclusion criteria were: (1) Studies focused on healthy volunteers with or without anxiety symptoms, or patients with a remitted or current PD; agoraphobia (AG); SAD; or generalised anxiety disorder (GAD). Comorbidity was allowed. (2) ATD was conducted measuring anxiety symptoms pre- and post-depletion. (3) The studies had either a within-subject or a between-subject design, with an ATD and sham intervention arm. In a within-subject design participants served as their own control. Animal studies, articles not presenting original data, consisting of abstracts only, or written in languages other than English or Dutch were excluded.

In accordance with the PRISMA guidelines ${ }^{21,22}$, study selection was conducted by two independent reviewers (SMES and NMB). Firstly, studies were assessed for eligibility based on titles and abstracts. Secondly, both reviewers independently assessed the method section of selected articles. Discrepancies were resolved through discussion.

\section{Data extraction and analysis}

For each article SMES and NMB independently extracted the following data: publication year, study design, presence and type of anxiety disorder, number of participants per intervention sequence, male/female ratio, used anxiety scales, outcomes on anxiety scales, use and type of challenge test and the corresponding outcomes. Discrepancies were resolved by referral to the original article. The primary outcome was the change in anxiety scores pre- and post-ATD compared to the sham intervention on the scales used in the original articles. The secondary outcome was the effect of a challenge test on anxiety scores.

\section{Quality assessment and publication bias}

Quality of studies was assessed using the Cochrane Collaboration tool as a guideline ${ }^{23}$. Assessed aspects were related to demographic and clinical characteristics, how ATD was conducted, and, if applicable, how the challenge test was conducted. RCB did the initial scoring and this was double-checked by NMB. In case of disagreement, consensus was reached through discussion.

Within the context of this, systematic review studies were rated to be of good quality when they used a randomised double-blind design, ATD was well executed and reported, and the relevant outcome measures were reported. When these items were insufficiently reported, the study was rated to be of medium quality. Studies which did not report the pre- and post-ATD anxiety scores nor the difference scores were rated to be of poor quality.

\section{ATD}

ATD is a technique to lower brain serotonin by reducing blood plasma tryptophan. In humans, most plasma tryptophan is bound to albumin, the remaining 'free tryptophan' can pass the blood-brain barrier into the central nervous system $^{13}$. In the brain the tryptophan hydroxylase enzyme 
first converts tryptophan into 5-hydroxytryptophan (5-HTP), which is then decarboxylated into 5-hydroxytryptamine (serotonin or 5-HT). The serotonin synthesis rate depends on the amount of free tryptophan in the blood, the amount of tryptophan passing the blood-brain barrier and the activity of the tryptophan hydroxylase enzyme. Along with tryptophan, five other large neutral amino acids (LNAAs) compete to pass the blood-brain barrier ${ }^{24}$. Maximum brain tryptophan depletion is achieved by combining a low tryptophan diet and an overnight fast with a drinkable tryptophan-deficient protein load containing large amounts of LNAAs. In healthy volunteers, concentrations of plasma tryptophan were highly correlated with cerebrospinal fluid levels of tryptophan, which suggests that ATD affects the serotonin turnover in humans in the central nervous system $^{25,26}$. Plasma tryptophan levels are lowest $5-7 \mathrm{~h}$ postingestion and hence, it is assumed that the effects of low levels of serotonin can then be studied ${ }^{26,27}$.

\section{Quality assessment of ATD}

The 'golden standard' for ATD consists of a low tryptophan diet $24 \mathrm{~h}$ before the test day, preceded by an overnight fast, which is extended throughout the day. For ATD a $100 \mathrm{~g}$ load of 15 amino acids, including other LNAAs but without tryptophan is given. This procedure tends to reduce tryptophan levels in the blood with approximately $80 \% 5-7 \mathrm{~h}$ post-ingestion ${ }^{26,27}$ and is considered high quality ATD. Reduction of plasma tryptophan levels is determined with a blood test.

In the selected studies, the quality of ATD was determined based on the description of the plasma tryptophan levels (free or total [free + bound to albumin]) before and after depletion and on the ratio between plasma tryptophan and other LNAAs. The percentage of change was calculated based on the reported tryptophan levels or LNAA ratio. In this study, ATD was defined to be of sufficient quality when plasma tryptophan decreased by at least $50 \%$. There is, however, still debate on when ATD can be considered to be of 'good' quality ${ }^{28}$.

\section{Results}

\section{Literature search}

The search in PubMed, Embase and PsychInfo resulted in 1609 unique records; of these, 1583 were excluded based on their title and abstract, resulting in 26 records for full-text screening (Fig. 1). Based on the inclusion criteria, 17 unique studies were identified with anxiety as outcome measure ${ }^{29-45}$. Cross referencing of the included articles resulted in the additional inclusion of four articles ${ }^{46-49}$. This systematic review thus contains 21 studies.

\section{Study characteristics}

Table 1 provides an overview of the characteristics of the 21 included studies. The studies were published between 1987 and 2012, and consisted of 16 withinsubject designs and 5 between-subject designs. The sample size ranged from 8 to 80 . Eleven studies investigated healthy volunteers, six remitted patients, two current patients and two both healthy volunteers and current patients. Investigated anxiety disorders were $\operatorname{GAD}(n=1)$, $\operatorname{SAD}(n=2)$ and $\operatorname{PD}(n=8)$.

Ten different challenge tests to provoke anxiety symptoms were used in 17 studies. Studies with healthy volunteers used tetrapeptide central cholecystokinin receptor agonist (CCK-4), 5-35\% carbon dioxide $\left(\mathrm{CO}_{2}\right)$, reaction time task, simulated public speaking (SPS), startle paradigm and yohimbine. Studies with PD patients used CCK-4, 5-35\% $\mathrm{CO}_{2}$ and flumazenil. Both studies with SAD patients used an autobiographic script and the study with GAD patients used 7.5\% $\mathrm{CO}_{2}$ for at least $12 \mathrm{~min}$.

To assess anxiety 18 different outcome measures were used. The most commonly used scales were anxiety visual analogue scales (VAS-A) with scale end-points that varied between studies $(n=11)$, the state part of the State Trait Anxiety Inventory (STAI-S) $(n=11)$ and the Profile of Mood States (POMS) $(n=6)$. Due to the variety in included populations, the use of different challenge tests and the broad range of outcome measures the studies were considered too heterogeneous to be pooled for meta-analysis.

\section{Healthy volunteers}

Thirteen studies were conducted in healthy volunteers $(n=350)$. Based on ten studies there is little evidence that ATD alone increased anxiety in healthy volunteers. Two studies reported a significant increase in anxiety following ATD, but the findings were inconsistent with the other anxiety measures used ${ }^{41,46}$. None of the other studies found a significant effect of ATD alone on anxiety $^{30,35-37,44,47-49}$.

Ten studies used a challenge test. The effect of the challenge test alone on anxiety without ATD was determined by looking at the results of the sham depletion, unless the study reported the effects of the challenge test separately. The challenge test alone increased anxiety in six studies ${ }^{35,40-42,44,47}$, although most studies did not report whether the increase was statistically significant. In two studies the challenge test alone resulted in an increase on some anxiety measures, but not on others ${ }^{30,37}$. Two studies did not report the scores on the anxiety measures, the effect of the challenge test alone is thus unknown ${ }^{36,39}$.

The $5 \% \mathrm{CO}_{2}$ challenge, reaction time task, yohimbine or CCK-4 did not increase anxiety following ATD ${ }^{35,37,39,40,47}$ compared to the challenge without ATD. Mixed results were found for the $35 \% \mathrm{CO}_{2}$ challenge, SPS and startle paradigm $^{30,36,41,42,44}$.

Studies in healthy volunteers are heterogeneous with respect to the number of outcome measures $(n=17)$ and 


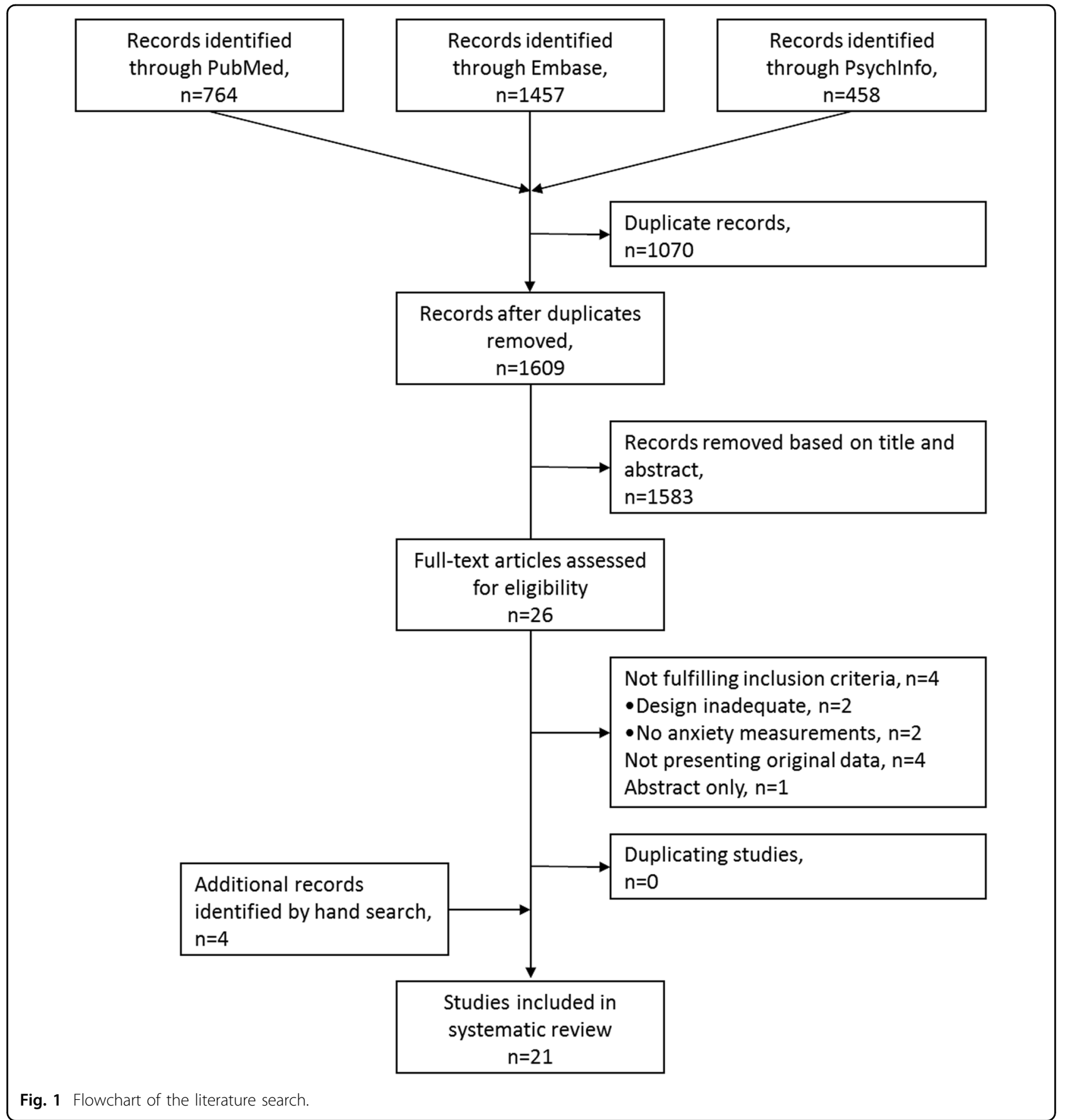

challenge tests $(n=7)$ used. In healthy volunteers neither ATD alone nor ATD with a challenge test consistently increased anxiety compared to sham depletion or sham depletion with a challenge test, respectively.

\section{Remitted patients}

Six studies were conducted in remitted patients $(n=$ 93). Patients were treated with cognitive behavioural therapy $(\mathrm{CBT})(n=1)^{38}$, with either CBT or a selective serotonin reuptake inhibitor (SSRI) $(n=1)^{45}$, or with SSRIs $(n=4)^{29,31-33}$. Five studies reported the effects of ATD alone. In three studies there were no indications that ATD increased anxiety ${ }^{29,38,45}$, and two studies reported mixed results ${ }^{32,33}$, with significant results on some but not on other anxiety measures.

In all six studies the challenge test alone increased anxiety, although it is unknown whether this increase was statistically significant. 


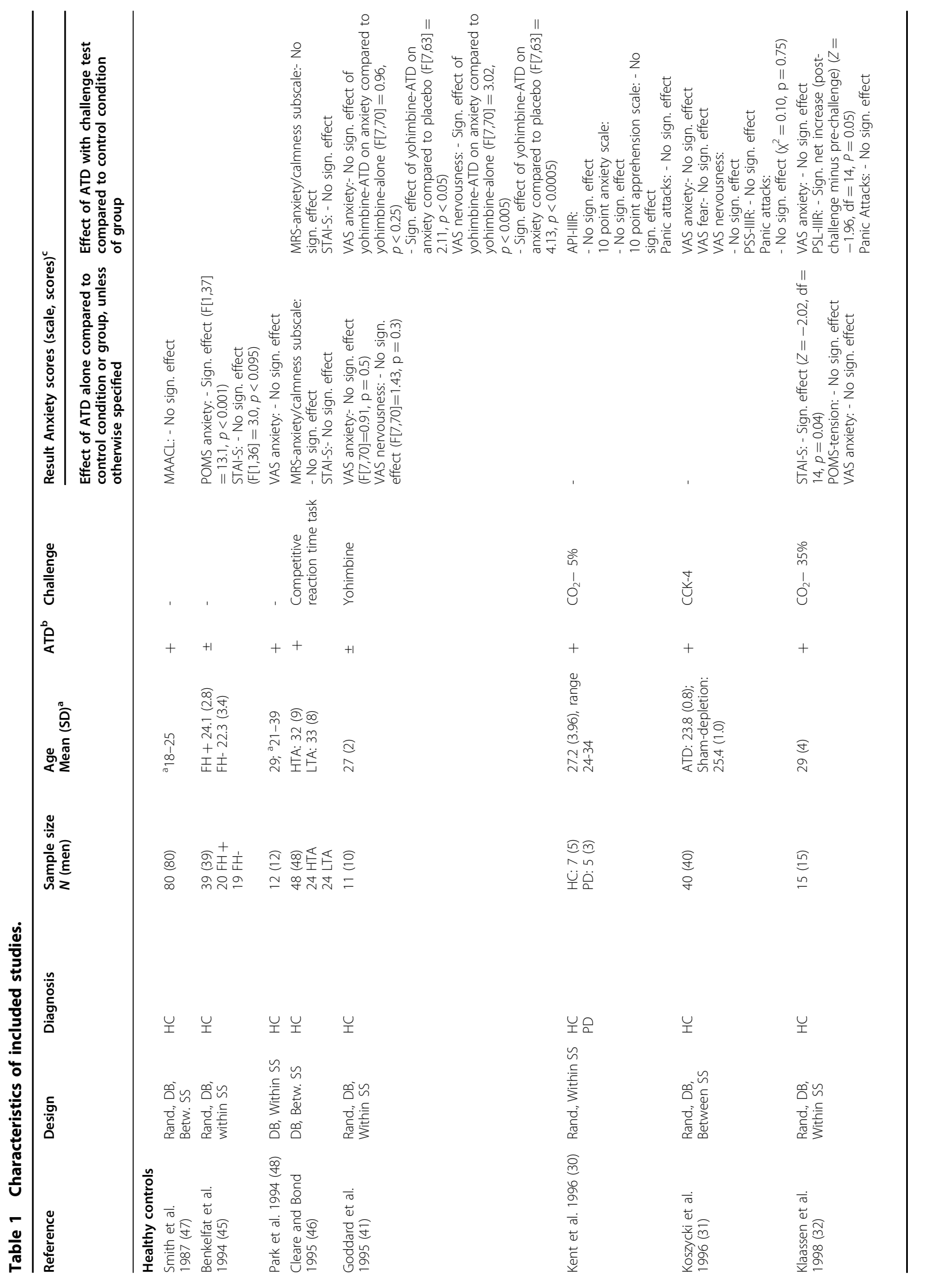




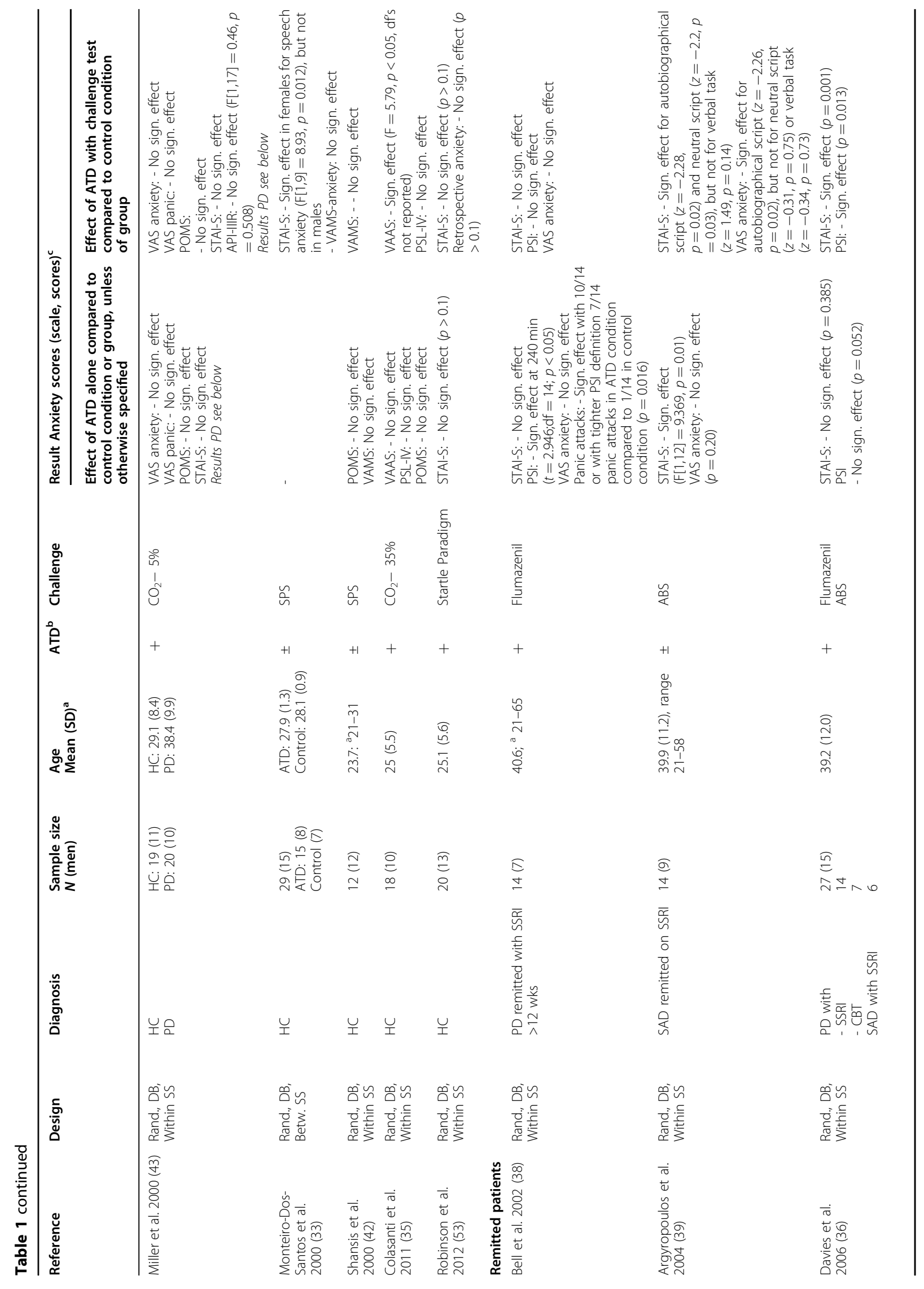




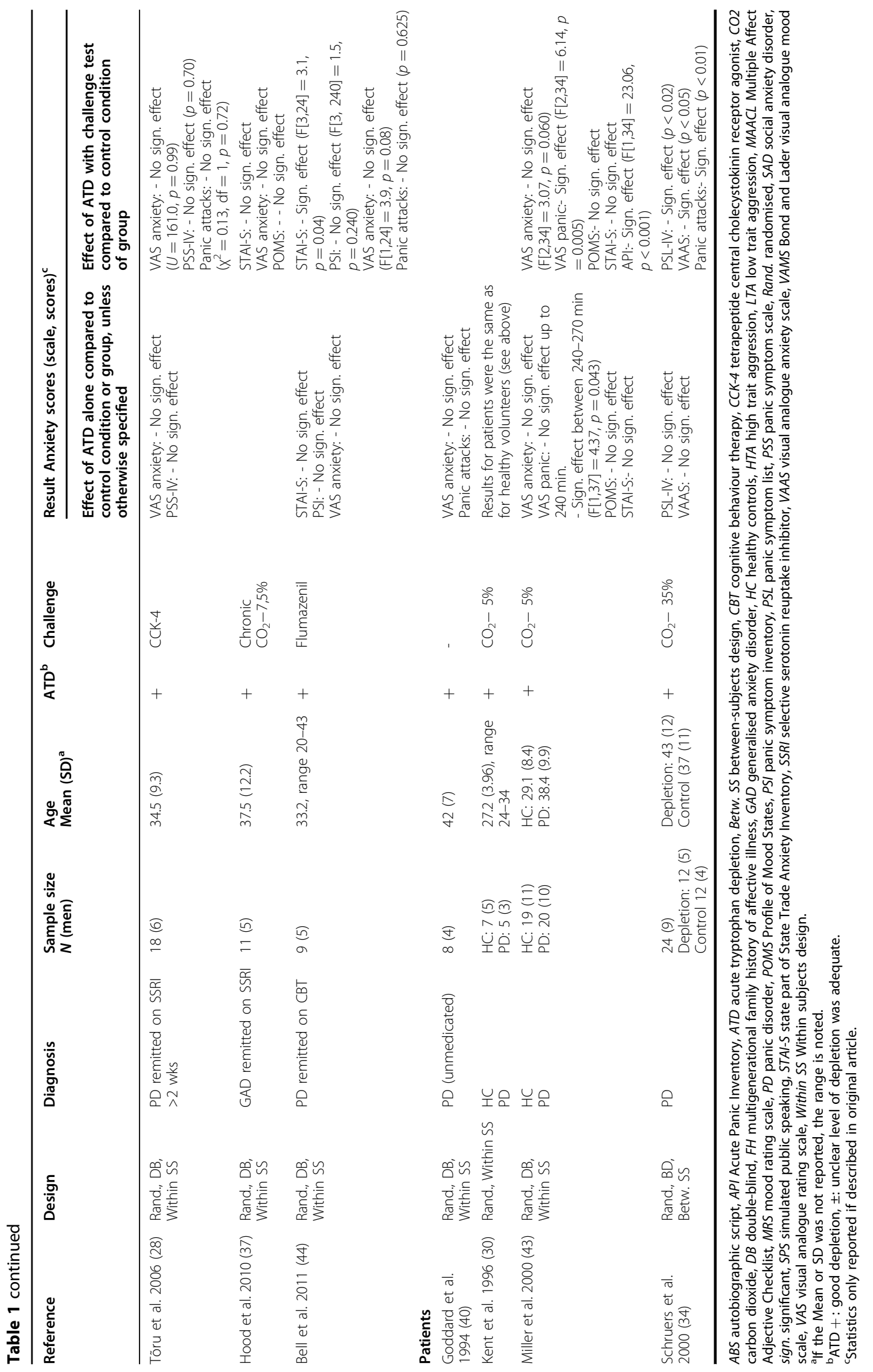


The results of the challenge test following ATD were mixed. There were no indications that $7.5 \% \mathrm{CO}_{2}$ or CCK4 increased anxiety in remitted GAD or PD patients ${ }^{29,31}$. Flumazenil was used to provoke panic symptoms in remitted PD patients with mixed results ${ }^{32,38,45}$ : two studies used the same anxiety measures ${ }^{32,38}$ with three out of four measures providing no indications for increased anxiety and the significant measure differed between studies. In the third study flumazenil with ATD did consistently increase anxiety ${ }^{45}$. Results of this study are difficult to interpret, as both PD and SAD patients were included. They received flumazenil or an autobiographical script respectively and the results were not reported per challenge test. Another study using an autobiographical script found that the challenge did significantly increase anxiety after ATD in remitted SAD patients ${ }^{33}$.

Studies in patients with a remitted anxiety disorder are heterogeneous in terms of the included anxiety disorder $(n=3)$, the used outcome measures $(n=6)$ and challenge tests $(n=4)$. In remitted patients ATD alone did not consistently increase anxiety and the use of challenge tests provided mixed results.

\section{Current patients}

Four studies were conducted in current PD patients $(n=57)$. Three studies reported the effects of ATD alone: in two studies anxiety was not increased ${ }^{34,43}$ and in one study results were $\operatorname{mixed}^{37}$.

Three studies used a challenge test. In two studies the challenge test alone increased anxiety ${ }^{37,43}$, although it is unknown whether this was statistically significant, and in one study the effect of the challenge alone is unknown as no anxiety scores were reported ${ }^{39}$.

Two studies used a $5 \% \mathrm{CO}_{2}$ challenge test after ATD, in one study no effect on anxiety was found ${ }^{39}$ and in the other results were mixed ${ }^{37}$. A $35 \% \mathrm{CO}_{2}$ challenge did result in a significant consistent increase in anxiety in current PD patients ${ }^{43}$.

Studies in current PD patients were heterogeneous in terms of outcome measures $(n=8)$. In current PD patients ATD alone did not increase anxiety. The use of a $35 \% \mathrm{CO}_{2}$ challenge test after ATD did significantly increase anxiety, but this was not found for lower $\mathrm{CO}_{2}$ concentrations.

\section{Quality assessment and publication bias}

In twelve studies a low tryptophan diet $24 \mathrm{~h}$ before ATD was included ${ }^{29-31,33,34,36-40,42,46}$, in three studies no diet was included ${ }^{35,47,48}$ and in five studies this was not reported $^{41,43-45,49}$. The exact content of the diet varied between studies. Fifteen studies included an overnight fast $^{29,31-33,35-38,40,41,43,46-49}$, but the duration of the fast varied. For the remaining studies it was unclear whether an overnight fast was required ${ }^{30,34,39,42,44,45}$. Blood tests showed that for total tryptophan the depletion ranged between 75.5-89.2\%, for free tryptophan between $58.3-86.7 \%$ and the LNAA ratio between $61.35-92.7 \%$. Studies measured the effect of ATD and the challenge test 4 to $7 \mathrm{~h}$ post-ingestion.

The quality assessment is reported in Table 2 . In all studies except one ${ }^{31}$ bias may have affected the outcomes. First, in three studies it was unclear how diagnostic status was determined $^{47-49}$ and in four studies no structured clinical interview was used ${ }^{34,35,42,45}$. Second, in two studies there were discrepancies between the reported and calculated percentages of change in plasma tryptophan levels ${ }^{33,46}$ and in three studies it was only reported that there was a significant difference between conditions, but the percentage change was not reported ${ }^{35,36,42}$. Third, in twelve studies $^{29,30,32-34,37,40,44,45,47-49}$ there is a high risk for attrition bias as dropouts were not reported or the number of dropouts was high $(>15 \%)$ without it being clear whether dropout was selective. Fourth, ten studies ${ }^{30,32-36,38,40,43,46}$ did not correct for multiple testing, though multiple comparisons were made using the anxiety and mood scales. Fifth, in eleven studies $29,32,34-39,41,44,47$ it was reported that there was a (non-)significant difference in anxiety and/or mood scores, but the pre-post test scores or difference scores were missing. Sixth, selection bias may be present in three stu$\operatorname{dies}^{42,47,49}$, as it was not clear whether participants were randomised. Finally, in one study ${ }^{39}$ there is a risk for detection bias as it is unclear if the study was conducted in a double-blind fashion. Overall, six studies were judged to be of 'good' quality because no major biases were present, five studies judged to be of 'medium' quality and ten studies of 'poor' quality.

\section{Discussion}

This systematic review aimed to investigate whether, in line with depression, ATD could reveal vulnerability for developing an anxiety disorder. In addition, we examined whether challenging anxiety modifies anxiety after ATD. Only 21 studies could be identified. The included studies were mostly of limited quality and heterogeneous in terms of included populations, challenge tests and outcome measures. As in depression ${ }^{14}$, anxiety did not consistently increase after ATD alone in healthy volunteers and current patients. Against expectations, there was also no consistent anxious response in remitted patients. With a challenge test, anxiety did not consistently increase in healthy volunteers, which is in line with their limited vulnerability for onset of anxiety disorders. Contrasting our hypothesis, although at risk ${ }^{6}$, a challenge test after ATD did not provoke anxiety in remitted patients. Only in current PD patients a $35 \% \mathrm{CO}_{2}$ test resulted in a consistent increase in anxiety on all measures after $\mathrm{ATD}^{43}$. This is possibly related to the suffocation alarm function PD patients being oversensitive. When challenged with 


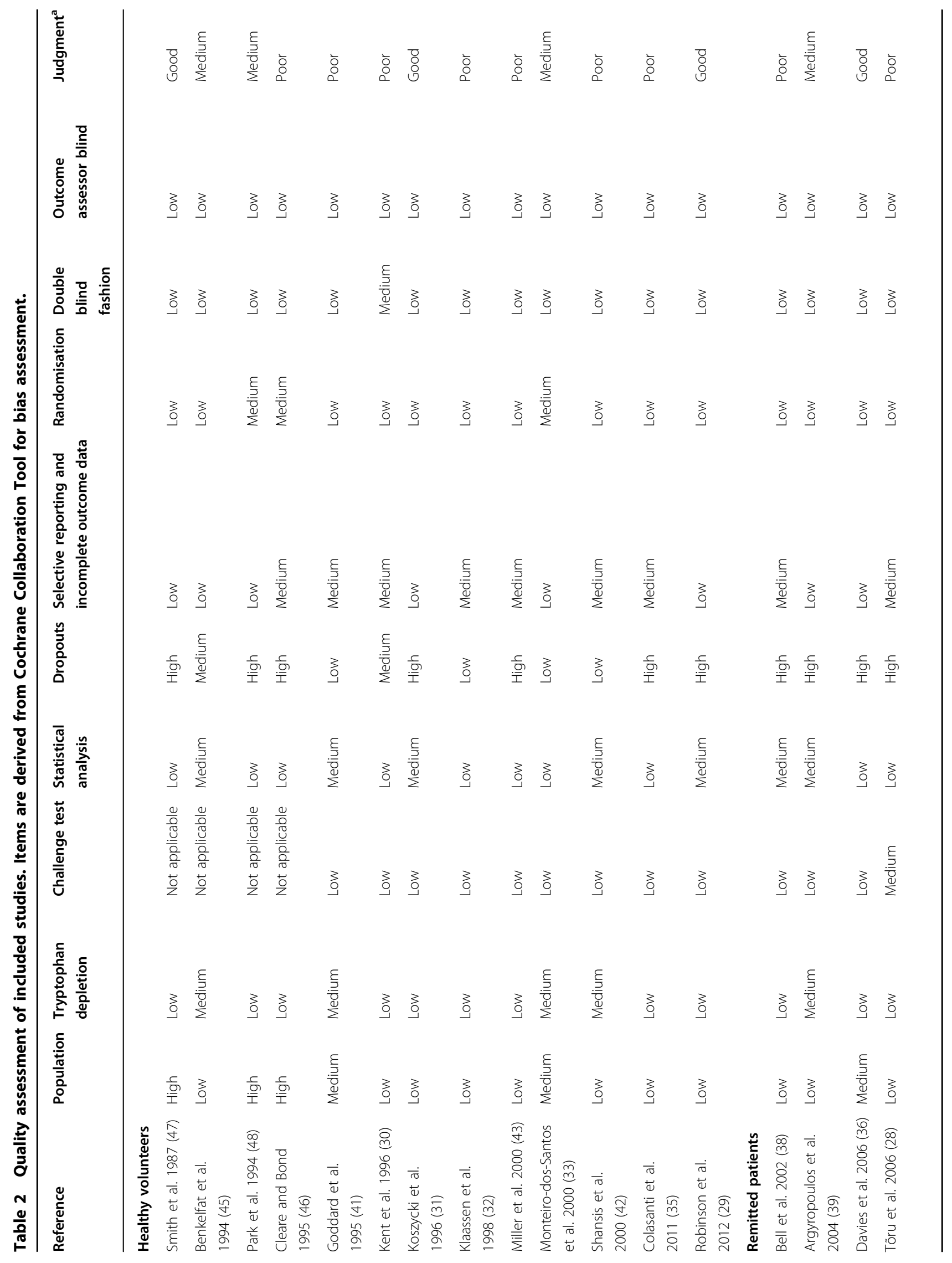




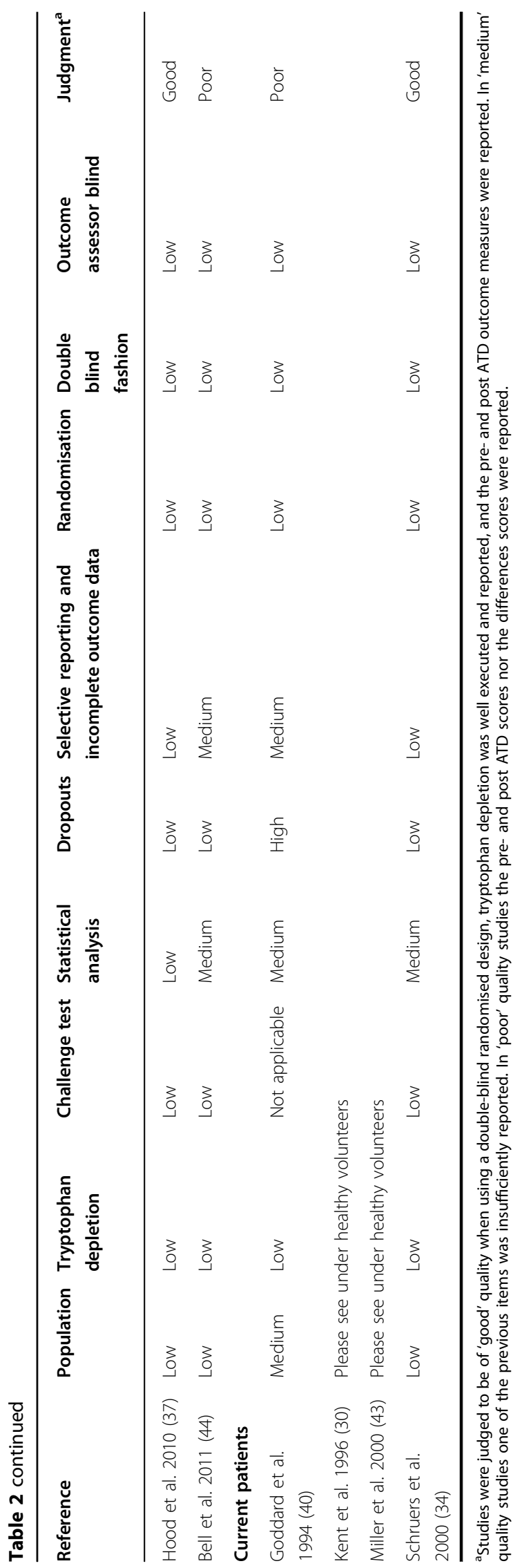

$\mathrm{CO}_{2}$ PD patients have an increased brainstem response compared to controls and the brainstem response is positively correlated with physical panic attack symptoms ${ }^{50}$. To what extend this is related to the underlying pathology is questionable, since patients with bilateral amygdalae lesions also respond to a $\mathrm{CO}_{2}$ challenge with panic and fear ${ }^{51}$. Other studies in current PD patients used a 5\% concentration of $\mathrm{CO}_{2}{ }^{37,39}$ and did not show this result. Currently there is little evidence that ATD can reveal vulnerability in anxiety disorders, which contrasts with the findings for depression.

In addition to dysregulations in the serotonin system $^{11}$, several other factors also contribute to the pathogenesis of anxiety disorders, including genetic, psychological and social factors (e.g. $\left.{ }^{52-56}\right)$. Studies in this systematic review were generally not controlled for such factors, while these factors could affect the outcomes of the studies. Variability in these factors among participants might explain why no effect of ATD on anxiety was observed. The serotonin system also plays a key role in depressive disorders ${ }^{11,17}$. In research for depression the impact of such factors was shown. It was for example previously found that the serotonin transporter genotype modulates the response to $\mathrm{ATD}^{57-59}$. Patients with homozygous long allele variants of the serotonin transporter gene SLC6A4 were generally more likely to respond with a depressed mood to ATD than patients with a homozygous short allele of this gene ${ }^{57-59}$. However, most ATD studies in depression did not control for factors that could have affected the outcomes, but nevertheless did find an effect of ATD on mood. It is therefore surprising that we found no indications for ATD revealing vulnerability in anxiety, in particular in remitted patients. To increase our understanding, we are thus in need of high quality studies with large sample sizes which do control for other factors, like genetic factors, that could affect the response to ATD.

A potential explanation for the lack of findings in remitted anxiety disorders is Deakin and Graeff's theory that serotonin mediates the response to aversive stimuli ${ }^{60}$. This theory assumes that the serotonin system is an anticipatory system to distal threats which influences the emotions fear and anxiety differentially: fear-related responses are inhibited from activating too early, while serotonin can facilitate anxiety responses to aversive stimuli ${ }^{60}$. ATD does not seem to result in a response in fearrelated disorders ${ }^{61}$. Most anxiety disorders belong to the 'fear' spectrum, namely SAD, specific phobia, AP and PD, in which anxiety mostly increases in feared 
situations. This is in contrast to GAD which is, together with depressive disorders, classified on the 'anxiety-misery' spectrum $^{62}$, in which anxiety or a negative mood is present most of the time. Given this subdivision, findings for GAD might be more similar to the findings for depressive disorders than for the fear-spectrum anxiety disorders. Only one small study included patients with remitted GAD $(n=11)^{31}$ and no significant effects were found. Given that of the remitted depressed patients $50-60 \%$ responds with a negative mood to $\mathrm{ATD}^{63}$, further investigation of GAD in larger populations may be of interest.

Alternatively, the timeframe for response to ATD for anxiety disorders might be later than for depressive disorders. This is congruent with the timing of the evaluation of the effect of antidepressant treatment, which according to the guidelines is for depressive disorders between 4 and 6 weeks and for anxiety disorders between 6 and 12 weeks $^{64-68}$. This difference in response latency may suggest a different involvement of the serotonergic system in these disorders or different interactions with other neurotransmitter systems. Possibly the timing of the evaluation of response needs to be later for anxiety than for depression. Instead, an anxious response may take longer to develop, which may require longer tryptophan depletion than necessary for a mood response. This remains to be investigated.

In depression, studies do not use a challenge test ${ }^{14}$, while in this systematic review 17 of 21 studies in anxiety used such a test. An explanation could be that, in depression, a negative mood is present most of the time, while anxiety generally increases in specific situations. Even when a challenge test is used, results showed no consistent effect of ATD on anxiety.

The challenge test may however induce ceiling effects and as a result no additional effect of ATD can be detected. For example, in healthy volunteers in whom ATD was combined with a yohimbine challenge, their anxious response was significantly increased after ATD with yohimbine compared to placebo, but not in comparison to yohimbine alone $\mathrm{e}^{35}$.

\section{Strengths and limitations}

A strength of this study is its systematic approach summarising probably all published ATD studies including anxiety measures. This study provides an overview of the published ATD research in anxiety, providing insight in the gaps of the current knowledge. Findings should be interpreted in context of the following limitations.

First, the selected studies showed heterogeneity. Due to the inclusion of different populations, anxiety disorders, challenge tests and outcome measures, results are difficult to compare and meta-analysis is precluded. The variability in the execution of ATD could also lead to false-positive/ negative results and hinders comparison between studies.

Second, many studies were of poor quality. Mostly because the scores on outcome measures of anxiety were not reported. Furthermore, it could not always be determined how the diagnostic status was assessed, whether dropouts were selective and whether randomisation had taken place.

Third, several studies found a significant increase on one anxiety measurement ${ }^{32,33,37,38,41,42,44,45}$, but results were often not corrected for multiple testing which could result in false positive findings. Furthermore, the measures that showed an effect differed between studies and different results have been observed between studies using the same measures in similar populations with a similar challenge test.

Fourth, the timing of the measures varied between studies. For example, post-ingestion and challenge test measures were taken $4-7 \mathrm{~h}$ post-ingestion. This could have affected the results, as the peak of tryptophan depletion is $5-7 \mathrm{~h}$ post-ingestion ${ }^{26,27}$.

Fifth, the sample sizes of the included studies were small, possibly resulting in underpowered studies and therefore lack of significant results. Although studies in depressive disorders had similar sample sizes, for anxiety fewer studies have been conducted (21 studies up to April 2019 versus 76 studies up to 2007). Patterns may thus not be elucidated due to the relatively low number of studies and the observed heterogeneity.

Sixth, results are mostly described on group level. Similar to depressive disorders it could be that only specific groups of participants respond with anxiety (e.g. ${ }^{14,57}$ ) and that this remains unobserved in the group effects.

Seventh, it remains to be determined which challenge tests are suitable for inducing anxiety after ATD. Challenge tests have been used which correspond with the characteristics of the disorder. It needs investigating to what extend these challenge tests result in ceiling effects and which challenge tests are most suitable to be used in healthy volunteers.

\section{Conclusion}

This systematic review showed that ATD research in anxiety disorders is heterogeneous, often of poor quality, and few studies have been conducted. Unlike in depressive disorders, ATD does not seem to be able to function as a marker of vulnerability for developing anxiety disorders. In anxiety disorders there is, thus, a need for good quality studies with large samples, which do control for other factors that could affect the response to ATD, before more definite conclusion can be drawn about lack of suitability of ATD as a marker for vulnerability in anxiety disorders. 


\section{Conflict of interest}

The authors declare that they have no conflict of interest.

\section{Publisher's note}

Springer Nature remains neutral with regard to jurisdictional claims in published maps and institutional affiliations.

Supplementary information The online version contains supplementary material available at https://doi.org/10.1038/s41398-021-01219-8.

Received: 14 February 2020 Revised: 30 October 2020 Accepted: 16 November 2020

Published online: 11 February 2021

\section{References}

1. Scholten, W. D. et al. Diagnostic instability of recurrence and the impact on recurrence rates in depressive and anxiety disorders. J. Affect Disord. 195 185-190 (2016).

2. Batelaan N. M., Rhebergen D., Spinhoven P., van Balkom AJLM, Penninx BWJH. Two-year course trajectories of anxiety disorders: do DSM classifications matter? J. Clin. Psychiatry. 75, 985-993 (2014)

3. Spinhoven, P. et al. Prediction of 6-yr symptom course trajectories of anxiety disorders by diagnostic, clinical and psychological variables. J. Anxiety Disord. 44, 92-101 (2016).

4. Penninx, B. W. J. H. et al. Two-year course of depressive and anxiety disorders: results from the Netherlands Study of Depression and Anxiety (NESDA). J. Affect Disord. 133, 76-85 (2011).

5. De Graaf, R., Ten Have, M., Tuithof, M. \& Van Dorsselaer, S. First-incidence of DSM-IV mood, anxiety and substance use disorders and its determinants: results from the Netherlands Mental Health Survey and Incidence Study-2. J. Affect Disord. 149, 100-107 (2013).

6. Scholten, W. D. et al. Recurrence of anxiety disorders and its predictors. J. Affect Disord. 147, 180-185 (2013).

7. Hek, K. et al. Anxiety disorders and salivary cortisol levels in older adults: a population-based study. Psychoneuroendocrinology 38, 300-305 (2013).

8. Vreeburg, S. A. et al. Salivary cortisol levels in persons with and without different anxiety disorders. Psychosom. Med. 72, 340-347 (2010).

9. Vogelzangs, N., Beekman, A. T. F., de Jonge, P. \& Penninx, B. W. J. H. Anxiety disorders and inflammation in a large adult cohort. Transl. Psychiatry 3, e249 (2013).

10. O'Donovan, A. et al. Clinical anxiety, cortisol and interleukin-6: evidence for specificity in emotion-biology relationships. Brain Behav. Immun. 24, 1074-1077 (2010)

11. Ressler K. J., Nemeroff C. B. Role of serotonergic and noradrenergic systems in the pathophysiology of depression and anxiety disorders. Depress Anxiety 12, 2-19 (2000).

12. van Donkelaar, E. L. et al. Mechanism of acute tryptophan depletion: is it only serotonin? Mol. Psychiatry 16, 695-713 (2011).

13. Hood, S. D., Bell, C. J. \& Nutt, D. J. Acute tryptophan depletion. Part I: rationale and methodology. Aust. N. Z. J. Psychiatry 39, 558-564 (2005).

14. Ruhé, H. G., Mason, N. S. \& Schene, A. H. Mood is indirectly related to serotonin, norepinephrine and dopamine levels in humans: a meta-analysis of monoamine depletion studies. Mol. Psychiatry 12, 331-359 (2007).

15. Booij, L. Van der Does A. J. W., \& Riedel, W. J. Monoamine depletion in psychiatric and healthy populations: review. Mol. Psychiatry 8, 951-973 (2003).

16. Bell, C. J., Hood, S. D. \& Nutt, D. J. Acute tryptophan depletion. Part II: clinical effects and implications. Aust. N. Z. J. Psychiatry 39, 565-574 (2005).

17. Gordon, J. A. \& Hen, R. The serotonergic system and anxiety. NeuroMolecular Med. 5, 27-40 (2004).

18. Frick, A. et al. Serotonin synthesis and reuptake in social anxiety disorder. JAMA Psychiatry 72, E1-E9 (2015).

19. Baldwin, D. S. et al. Evidence-based pharmacological treatment of anxiety disorders, post-traumatic stress disorder and obsessive-compulsive disorder: a revision of the 2005 guidelines from the British Association for Psychopharmacology. J. Psychopharmacol. 28, 403-439 (2014).
20. Bandelow, B. et al. Guidelines for the pharmacological treatment of anxiety disorders, obsessive-compulsive disorder and posttraumatic stress disorder in primary care. Int J. Psychiatry Clin. Pract. 16, 77-84 (2012).

21. Liberati, A. et al. The PRISMA statement for reporting systematic reviews and meta-analyses of studies that evaluate health care interventions: explanation and elaboration. J. Clin. Epidemiol. 62, e1-e34 (2009).

22. Moher, D. et al. Preferred reporting items for systematic review and metaanalysis protocols (PRISMA-P) 2015 statement. Syst. Rev. 4, 1 (2015).

23. Higgins, J. P. T. et al. The Cochrane Collaboration's tool for assessing risk of bias in randomised trials. BMJ 343, d5928-d5928 (2011).

24. Reilly, J. G., Mctavish, S. F. B. \& Young, A. H. Rapid depletion of plasma tryptophan: a review of studies and experimental methodology. J. Psychopharmacol. 11, 381-392 (1997)

25. Carpenter, L. L. et al. Tryptophan depletion during continuous CSF sampling in healthy human subjects. Neuropsychopharmacology 19, 26-35 (1998).

26. Williams, W. A., Shoaf, S. E., Hommer, D., Rawlings, R. \& Linnoila, M. Effects of acute tryptophan depletion on plasma and cerebrospinal fluid tryptophan and 5-hydroxyindoleacetic acid in normal volunteers. J. Neurochem. 72 1641-1647 (1999).

27. Delgado, P. L. Serotonin function and the mechanism of antidepressant action. Arch. Gen. Psychiatry 47, 411 (1990).

28. Young, S. N. Acute tryptophan depletion in humans: a review of theoretical, practical and ethical aspects. J. Psychiatry Neurosci. 38, 294-305 (2013).

29. Tõru, I., Shlik, J., Maron, E., Vasar, V. \& Nutt, D. J. Tryptophan depletion does not modify response to CCK-4 challenge in patients with panic disorder after treatment with citalopram. Psychopharmacol. 186, 107-112 (2006).

30. Robinson, O. J., Overstreet, C., Allen, P. S., Pine, D. S. \& Grillon, C. Acute tryptophan depletion increases translational indices of anxiety but not fear: serotonergic modulation of the bed nucleus of the stria terminalis? Neuropsychopharmacology 37, 1963-1971 (2012).

31. Hood, S. D. et al. Effects of acute tryptophan depletion in serotonin reuptake inhibitor-remitted patients with generalized anxiety disorder. Psychopharma col. 208, 223-232 (2010)

32. Bell, C. et al. Does 5-HT restrain panic? A tryptophan depletion study in panic disorder patients recovered on paroxetine. J. Psychopharmacol. 16, 5-14 (2002).

33. Argyropoulos, S. V. et al. Tryptophan depletion reverses the therapeutic effect of selective serotonin reuptake inhibitors in social anxiety disorder. Biol. Psychiatry 56, 503-509 (2004).

34. Goddard, A. W. et al. Effects of tryptophan depletion in panic disorder. Biol. Psychiatry 36, 775-777 (1994).

35. Goddard, A. W. et al. Effects of tryptophan depletion on responses to yohimbine in healthy human subjects. Biol. Psychiatry 38, 74-85 (1995).

36. Shansis, F. M. et al. Behavioural effects of acute tryptophan depletion in healthy male volunteers. J. Psychopharmacol. 14, 157-163 (2000).

37. Miller, H. E. J., Deakin, J. F. W. \& Anderson, I. M. Effect of acute tryptophan depletion on $\mathrm{CO}_{2}$-induced anxiety in patients with panic disorder and normal volunteers. Br. J. Psychiatry 176, 182188 (2000).

38. Bell, C. et al. Rapid tryptophan depletion following cognitive behavioural therapy for panic disorder. Psychopharmacol. 213, 593-602 (2011).

39. Kent, J. M. et al. Ventilatory effects of tryptophan depletion in panic disorder: a preliminary report. Psychiatry Res. 64, 83-90 (1996).

40. Koszycki, D., Zabarcko, R. M., Le Melledo, J.-M., Young, S. \& Bradwejn, J. Effect of acute tryptophan depletion on behavioral, cardiovascular, and hormonal sensitivity to cholecystokinin tetrapeptide challenge in healthy volunteers. Biol. Psychiatry 40, 648-655 (1996).

41. Klaassen, T., Klumperbeek, J., Deutz, N. E. P., van Praag, H. M. \& Griez, E. Effects of tryptophan depletion on carbon dioxide provoked panic in panic disorder patients. Psychiatry Res. 77, 167-174 (1998).

42. Monteiro-Dos-Santos, P. C. et al. Effects of tryptophan depletion on anxiety induced by simulated public speaking. Braz. J. Med. Biol. Res. 33, 581-587 (2000).

43. Schruers, K. et al. Effects of tryptophan depletion on carbon dioxide provoked panic in panic disorder patients. Psychiatry Res. 93, 179-187 (2000).

44. Colasanti, A. et al. Effects of tryptophan depletion and tryptophan loading on the affective response to high-dose $\mathrm{CO} 2$ challenge in healthy volunteers. Psychopharmacol. 215, 739-748 (2011).

45. Davies, S. J. C. et al. Depleting serotonin enhances both cardiovascular and psychological stress reactivity in recovered patients with anxiety disorders. J. Clin. Psychopharmacol. 26, 414-418 (2006). 
46. Benkelfat, C., Ellenbogen, M. A., Dean, P., Palmour, R. M. \& Young, S. N. Moodlowering effect of tryptophan depletion. Arch. Gen. Psychiatry 51, 687-697 (1994).

47. Cleare, A. J. \& Bond, A. J. The effect of tryptophan depletion and enhancement on subjective and behavioural aggression in normal male subjects. Psychopharmacol. 118, 72-81 (1995).

48. Smith, S. E., Pihl, R. O., Young, S. N. \& Ervin, F. R. A test of possible cognitive and environmental influences on the mood lowering effect of tryptophan depletion in normal males. Psychopharmacol. 91, 451-457 (1987).

49. Park, S. B. et al. Tryptophan depletion in normal volunteers produces selective impairments in learning and memory. Neuropharmacology 33, 575-588 (1994).

50. Goossens, L. et al. Brainstem response to hypercapnia: a symptom provocation study into the pathophysiology of panic disorder. J. Psychopharmacol. 28, 449-456 (2014).

51. Feinstein, J. S. et al. Fear and panic in humans with bilateral amygdala damage. Nat. Neurosci. 16, 270-272 (2013).

52. Otowa, T. et al. Meta-analysis of genome-wide association studies of anxiety disorders. Mol. Psychiatry 21, 1391-1399 (2016).

53. Shimada-Sugimoto, M., Otowa, T. \& Hettema, J. M. Genetics of anxiety disorders: genetic epidemiological and molecular studies in humans. Psychiatry Clin. Neurosci. 69, 388-401 (2015).

54. Schmidt, N. B., Zvolensky, M. J. \& Maner, J. K. Anxiety sensitivity: prospective prediction of panic attacks and \{Axis\} \{l\} pathology. J. Psychiatr. Res. 40 691-699 (2006).

55. Burstein, M. \& Ginsburg, G. S. The effect of parental modeling of anxious behaviors and cognitions in school-aged children: an experimental pilot study. Behav. Res. Ther. 48, 506-515 (2010).

56. Muris, P., Steerneman, P., Merckelbach, H. \& Meesters, C. The role of parental fearfulness and modeling in children's fear. Behav. Res. Ther. 34, 265-268 (1996).

57. Moreno, F. A. et al. Association study of genotype by depressive response during acute tryptophan depletion in subjects recovered from major depression. Mol. Neuropsychiatry 1, 165-174 (2015).
58. Moreno, F. A. et al. Association between a serotonin transporter promoter region polymorphism and mood response during tryptophan depletion. $\mathrm{Mol}$ Psychiatry 7, 213-216 (2002).

59. Neumeister, A. et al. Differential effects of 5-HTTLPR genotypes on the behavioral and neural responses to tryptophan depletion in patients with major depression and controls. Arch. Gen. Psychiatry 63, 978 (2006)

60. Deakin, J. F. W. The origins of '5-HT and mechanisms of defence' by Deakin and Graeff: a personal perspective. J. Psychopharmacol. 27, 1084-1089 (2013).

61. Corchs, F. et al. Evidence for serotonin function as a neurochemical difference between fear and anxiety disorders in humans? J. Psychopharmacol. 29, 1061-1069 (2015).

62. Krueger, R. F. The structure of common mental disorders. Arch. Gen. Psychiatry 56, 921 (1999).

63. Booij, L. et al. Predictors of mood response to acute tryptophan depletion: a reanalysis. Neuropsychopharmacology 27, 884-885 (2002)

64. Bauer, M. et al. World Federation of Societies of Biological Psychiatry (WFSBP) guidelines for biological treatment of unipolar depressive disorders, part 1: update 2013 on the acute and continuation treatment of unipolar depressive disorders. World J. Biol. Psychiatry 14, 334-385 (2013).

65. Cleare, A. J. et al. Evidence-based guidelines for treating depressive disorders with antidepressants: a revision of the 2008 British Association for Psychopharmacology guidelines. J. Psychopharmacol. 29, 459-525 (2015).

66. National Institute for Health and Clinical Excellence. Generalised Anxiety Disorder and Panic Disorder (with or without agoraphobia) in Adults. Management in Primary, Secondary and Community Care (National Institute for Health and Care Excellence, 2011).

67. National Institute for Health and Clinical Excellence. Depression in Adults: Recognition and Management (National Institute for Health and Care Excellence, 2009).

68. National Institute for Health and Clinical Excellence. Social Anxiety Disorder: Recognition, Assessment and Treatment (National Institute for Health and Care Excellence, 2013). 